ASC Report No. 27/2007

\title{
On Semibounded Canonical Systems
}

Henrik Winkler, Harald Woracek 


\section{Most recent ASC Reports}

26/2007 Irena Rachunková, Gernot Pulverer, Ewa B. Weinmüller

A Unified Approach to Singular Problems Arising in the Membrane Theory

25/2007 Roberta Bosi, Jean Dolbeault, Maria J. Esteban

Estimates for the Optimal Constants in Multipolar Hardy Inequalities for Schrödinger and Dirac Operators

24/2007 Xavier Antoine, Anton Arnold, Christophe Besse, Matthias Ehrhardt, Achim Schädle

A Review of Transparent and Artificial Boundary Conditions Techniques for Linear and Nonlinear Schrödinger Equations

23/2007 Tino Eibner, Jens Markus Melenk

p-FEM Quadrature Error Analysis on Tetrahedra

22/2007 Stephan Gadau, Ansgar Jüngel

A 3D Mixed Finite-element Approximation of the Semiconductor Energytransport Equations

21/2007 Anton Arnold

Mathematical Properties of Quantum Evolution Equations

20/2007 Winfried Auzinger, Herbert Lehner, Ewa Weinmüller

Defect-based A-posteriori Error Estimation for Index-1 DAEs

19/2007 Georg Kitzhofer, Othmar Koch, Ewa Weinmüller

Pathfollowing for Essentially Singular Boundary Value Problems with Application to the Complex Ginzburg-Landau Equation

18/2007 Svatoslav Staněk, Gernot Pulverer, Ewa B. Weinmüller

Analysis and Numerical Simulation of Positive and Dead Core Solutions of Singular Two-point Boundary Value Problems

17/2007 Thorsten Sickenberger, Ewa Weinmüller, Renate Winkler

Local Error Estimates for Moderately Smooth Problems: Part II - SDEs and SDAEs with Small Noise

Institute for Analysis and Scientific Computing

Vienna University of Technology

Wiedner Hauptstraße 8-10

1040 Wien, Austria

E-Mail: admin@asc.tuwien.ac.at

WWW: http://www.asc.tuwien.ac.at

FAX: $\quad+43-1-58801-10196$

ISBN 978-3-902627-00-1

(C) Alle Rechte vorbehalten. Nachdruck nur mit Genehmigung des Autors.

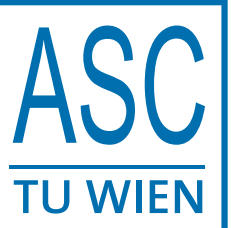




\title{
On semibounded canonical systems
}

\author{
Henrik Winkler and Harald Woracek
}

\begin{abstract}
We present two inverse spectral relations for canonical differential equations $J y^{\prime}(x)=-z H(x) y(x), x \in[0, L)$ : Denote by $Q_{H}$ the TitchmarshWeyl coefficient associated with this equation. We show: If the Hamiltonian $H$ is on some interval $[0, \epsilon)$ of the form

$$
H(x)=\left(\begin{array}{cc}
v(x)^{2} & v(x) \\
v(x) & 1
\end{array}\right),
$$

with a nondecreasing function $v$, then $\lim _{x \backslash 0} v(x)=\lim _{y \rightarrow+\infty} Q_{H}(i y)$. If $H$ is of the above form on some interval $[l, L)$, then $\lim _{x} \nearrow_{L} v(x)=\lim _{z} \nearrow_{0} Q_{H}(z)$. In particular, these results are applicable to semibounded canonical systems, or canonical systems with a finite number of negative eigenvalues, respectively.

Mathematics Subject Classification (2000). Primary 34A55, 47E05, 34B20; Secondary 34L05, 47B25.
\end{abstract}

Keywords. canonical (Hamiltonian) system, Titchmarsh-Weyl coefficient, Inverse spectral problem.

\section{Introduction}

A canonical (or Hamiltonian) system is an boundary value problem of the form

$$
J y^{\prime}(x)=-z H(x) y(x), x \in[0, L), \quad y_{1}(0)=0,
$$

where $L \in(0, \infty]$, and where $H$ is a function which takes real, symmetric and nonnegative $2 \times 2$-matrices as values, does not vanish on any set of positive measure, and belongs to $L_{l o c}^{1}([0, L))$. Moreover, $z$ is a complex parameter and

$$
J:=\left(\begin{array}{cc}
0 & -1 \\
1 & 0
\end{array}\right) .
$$

The first author was supported by the "Fond zur Förderung der wissenschaftlichen Forschung" (FWF,Austria), grant number P15540-N05. 
The function $H$ is called the Hamiltonian of the system (1.1). Canonical systems occur in mathematical physics and were intensively investigated, see e.g. [1], [3], $[4],[7],[8]$.

The condition

$$
\int_{0}^{L} \operatorname{trace} H(x) d x=+\infty
$$

plays a crucial role in the spectral theory of canonical systems. In fact, (1.2) says that the so-called Weyl's limit point case prevails. To a system (1.1) which satisifes (1.2) there is associated a function $Q_{H}(z)$, its Titchmarsh-Weyl coefficient, which belongs to the Nevanlinna class $\mathcal{N}$. This is the set of all functions $Q$ analytic on $\mathbb{C} \backslash \mathbb{R}, Q(\bar{z})=\overline{Q(z)}$, with $\operatorname{Im} Q(z) \geq 0$ for Im $z>0$. The Inverse Spectral Theorem of L.de Branges states that the assignment $H \mapsto Q_{H}$ yields, up to changes of scale, a bijection of the set of all Hamiltonians which satsify (1.2) onto $\mathcal{N} \cup\{\infty\}$.

Inverse spectral relations are statements which relate properties of $Q_{H}$ to preoperties of $H$. In this paper we establish two statements of this kind. We show that, if the Hamiltonian is on some interval $[0, \epsilon)$ of the form

$$
H(x)=\left(\begin{array}{cc}
v(x)^{2} & v(x) \\
v(x) & 1
\end{array}\right)
$$

where $v$ is nondecreasing, then $\lim _{x \backslash 0} v(x)=\lim _{y \rightarrow+\infty} Q_{H}(i y)$, cf. Theorem 3.3, and that, if $H$ is of the above form on some interval $[l, L)$, then $\lim _{x}{ }_{L} v(x)=$ $\lim _{z \succ 0} Q_{H}(z)$, cf. Theorem 3.9.

Our investigations are motivated by the study of semibounded canonical systems, that are systems with the property that their Titchmarsh-Weyl coefficient has an analytic continuation to some set of the form $\mathbb{C} \backslash[M, \infty)$, cf. Theorem 2.3, Corollary 3.5. Proofs are based on the theory of strings, cf. [15]. The statement in Corollary 3.5 also finds some application in the extension theory of symmetric relations, for, it shows a straightforward way to determine the Friedrichs extension in terms of the Hamiltonian, see [9], [11] and [23] for details.

In the preliminary Section 2 we set up our notation and recall some results which will be used later on. In Section 3 we prove and discuss our main results Theorem 3.3 and Theorem 3.9.

\section{Preliminaries}

\section{A. Nevanlinna functions}

By the Herglotz representation theorem, a Nevanlinna function $Q$ has an integral representation of the form

$$
Q(z)=b z+a+\int_{\mathbb{R}}\left(\frac{1}{\lambda-z}-\frac{\lambda}{1+\lambda^{2}}\right) d \sigma(\lambda),
$$

with $b \geq 0, a \in \mathbb{R}$, and a measure $\sigma$ satisfying $\int_{\mathbb{R}}\left(1+\lambda^{2}\right)^{-1} d \sigma(\lambda)<\infty$. Thereby $a, b$ and $\sigma$ are uniquely determined by $Q$. Many interesting subclasses of $\mathcal{N}$ can be 
defined, or characterized, in terms of $a, b$ and $\sigma$. In our context two subclasses will play an important role: the Kac class $\mathcal{N}_{1}$ and the Stieltjes class $\mathcal{S}$.

The Kac class $\mathcal{N}_{1}$ is defined as the set of all $Q \in \mathcal{N}$ with

$$
b=0, \int_{\mathbb{R}} \frac{d \sigma(\lambda)}{1+|\lambda|}<\infty .
$$

This means that $Q \in \mathcal{N}_{1}$ if and only if it can be represented as

$$
Q(z)=\alpha+\int_{\mathbb{R}} \frac{d \sigma(\lambda)}{\lambda-z}
$$

with some $\alpha \in \mathbb{R}$ and $\int_{\mathbb{R}}(1+|\lambda|)^{-1} d \sigma(\lambda)<\infty$. An analytic characterization of $\mathcal{N}_{1}$ was given in [13], see also [14, Theorem S1.3.1]: A Nevanlinna function $Q$ belongs to $\mathcal{N}_{1}$ if and only if

$$
\int_{1}^{\infty} \frac{\operatorname{Im} Q(i y)}{y} d y<\infty
$$

For a closer investigation of Kac classes and related subjects see also [2], [10] or [23].

The Stieltjes class $\mathcal{S}$ is defined as the set of all functions $Q$ which are analytic in $\mathbb{C} \backslash[0, \infty)$, satisfy $\operatorname{Im} Q(z) \geq 0, z \in \mathbb{C}^{+}$, and $Q(z) \geq 0, z \in(-\infty, 0)$. Clearly, $\mathcal{S} \subseteq \mathcal{N}$. The history of the class $\mathcal{S}$ goes back to some investigations of T.J.Stieltjes on the moment problem and continued fractions, cf. [19]. Also the class $\mathcal{S}$ can be characterized in various ways, cf. [14, Theorem S1.5.1, Lemma S1.5.1]. In fact, for a function $Q$ which is analytic in $\mathbb{C} \backslash[0, \infty)$ and satisfies $Q(\bar{z})=\overline{Q(z)}$, the following conditions are equivalent:

1. $Q \in \mathcal{S}$.

2. $Q \in \mathcal{N}_{1}$, supp $\sigma \subseteq[0, \infty)$, and the constant $\alpha$ in (2.2) is nonnegative.

3. $Q(z) \in \mathcal{N}$ and $z Q(z) \in \mathcal{N}$.

4. $z Q\left(z^{2}\right) \in \mathcal{N}$.

Further investigations and generalizations of the Stieltjes class can be found e.g. in [2], [5], or [16].

\section{B. Canonical systems}

Let us recall the construction of the Titchmarsh-Weyl coefficient associated to a Hamiltonian $H$ : Denote by

$$
W(x, z)=\left(\begin{array}{ll}
w_{11}(x, z) & w_{12}(x, z) \\
w_{21}(x, z) & w_{22}(x, z)
\end{array}\right), W(0, z)=I
$$

the transposed of the fundamental matrix solution of the system (1.1). That is, $W(x, z)$ is the unique solution of $\frac{\partial}{\partial x} W(x, z) J=z W(x, z) H(x), W(0, z)=I$. Then, since we assume that (1.2) holds, for each $\omega \in \mathcal{N} \cup\{\infty\}$ and $z \in \mathbb{C}^{+}$the limit

$$
Q_{H}(z):=\lim _{x \rightarrow L} \frac{w_{11}(x, z) \omega(z)+w_{12}(x, z)}{w_{21}(x, z) \omega(z)+w_{22}(x, z)}
$$


exists, is independent of $\omega$, and, as a function of $z$, belongs to $\mathcal{N} \cup\{\infty\}$, see e.g. [4]. This is the Titchmarsh-Weyl coefficient associated with $H$. The measure $\sigma_{H}$ in the integral representation (2.1) of $Q_{H}$ is called the spectral measure of $H$.

Two Hamiltonians $H_{1}$ on $\left[0, L_{1}\right)$ and $H_{2}$ on $\left[0, L_{2}\right)$ are said to be reparameterizations of each other, $H_{1} \sim H_{2}$, if there exists a strictly increasing bijection $\lambda$ of $\left[0, L_{1}\right)$ onto $\left[0, L_{2}\right)$ such that $H_{1}(x)=H_{2}(\lambda(x)) \lambda^{\prime}(x), x \in\left[0, L_{1}\right)$. It is easy to see that, if $H_{1} \sim H_{2}$, then $Q_{H_{1}}=Q_{H_{2}}$.

The basic inverse result of L.de Branges is, cf. [4], [20]:

Theorem 2.1 (Inverse Spectral Theorem). The assignment $H \mapsto Q_{H}$ sets up a bijection between the set of all Hamiltonians modulo $\sim$ and $\mathcal{N} \cup\{\infty\}$.

To illustrate the nature of inverse spectral relations, let us mention two results of this kind, which will also be of good use later on:

Remark 2.2 .

1. If we assume that trace $H(t) \equiv 1$, which can always be achieved by a suitable reparameterization, then the constant $b$ in the integral representation of $Q_{H}$ is the maximal number such that $\left.H\right|_{[0, b)}=\operatorname{diag}(1,0)$, cf. [15].

2. Let $\sigma$ be the measure in the integral representation of $Q_{H}$. Then

$$
\int_{0}^{L}(0,1) H(x)\left(\begin{array}{l}
0 \\
1
\end{array}\right) d x=\frac{1}{\sigma(\{0\})},
$$

where the right hand side is understood as $+\infty$ if $\sigma(\{0\})=0$. This fact was proved in [22, Theorem 2.2].

\section{Transformation of canonical systems}

We will employ two transformations of Hamiltonians. These, and others, were investigated in [21].

Let $H$ be a Hamiltonian defined on $[0, L)$. Then also

$$
\widehat{H}:=J H J^{T}
$$

is a Hamiltonian on $[0, L)$. Clearly $H$ and $\widehat{H}$ together do or do not satisfy $(1.2)$. The fundamental matrix $\widehat{W}$ corresponding to $\widehat{H}$ satisfies the relation $\widehat{W}=J W J^{T}$. Hence, by (2.4), we have

$$
Q_{\widehat{H}}(z)=-Q_{H}(z)^{-1}
$$

Let again $H$ be a Hamiltonian defined on $[0, L)$ and let $c \in \mathbb{R}$. Then also

$$
\widehat{H}:=C H C^{T},
$$

where $C:=\left(\begin{array}{ll}1 & c \\ 0 & 1\end{array}\right)$, is a Hamiltonian on $[0, L)$ and satisfies together with $H$ the condition (1.2). In this situation we have $Q_{\widehat{H}}(z)=Q_{H}(z)+c$. 


\section{Semibounded canonical systems}

One of the main objects of our studies are canonical systems whose spectral measure is semibounded from below. Recall the following result which was proved, in a slightly different formulation, in [22].

Theorem 2.3. Let $Q \in \mathcal{N}$ be a Nevanlinna function with $\inf \operatorname{supp} \sigma>-\infty$. Then there exists a number $L \in(0, \infty]$ and a nondecreasing and right-continuous function $\nu:[0, L) \rightarrow[0,+\infty)$ such that, with $v(x):=-\cot \nu(x), \nu(x) \notin \pi \mathbb{Z}$, the Hamiltonian

$$
H(x)= \begin{cases}\left(\begin{array}{cc}
v(x)^{2} & v(x) \\
v(x) & 1
\end{array}\right) & \text { if } \nu(x) \notin \pi \mathbb{Z} \\
\left(\begin{array}{ll}
1 & 0 \\
0 & 0
\end{array}\right) & \text { if } \nu(x) \in \pi \mathbb{Z}\end{cases}
$$

satsifies (1.2) and $Q_{H}=Q$. If $\nu$ is normalized in such a way that $\nu(0) \in[0, \pi)$ and $\nu(x)-\nu(x-)<\pi$, then $L$ and $\nu$ are unique.

The function $\nu$ (if normalized as above) is bounded if and only if $(-\infty, 0) \cap$ $\operatorname{supp} \sigma$ is finite. If $\nu(L-) / \pi \in \mathbb{N}$, then $Q$ has $n-1$ poles on $(-\infty, 0)$, otherwise the number of poles of $Q$ on $(-\infty, 0)$ is equal to the integer part of $\nu(L-) / \pi$.

It is not known to the authors whether or not the converse of this result holds. However, in a particular case a converse can be proved, cf. [22].

Theorem 2.4. Let $H$ be a Hamiltonian of the form (2.9), and assume that $\nu$ is bounded. Then, for the spectral measure $\sigma$ of $H$, we have inf $\operatorname{supp} \sigma>-\infty$.

Let $Q \in \mathcal{N}, \inf \operatorname{supp} \sigma>-\infty$, and let $\nu$ be as in Theorem 2.3. Then the constant $b$ in the integral representation (2.1) of $Q$ is determined by

$$
b=\sup (\{x \geq 0: \nu(x)=0\} \cup\{0\}) .
$$

Hence, if $b=0$, there exists a nonempty interval $(0, \epsilon)$, such that $\nu(x) \notin \pi \mathbb{Z}$, $x \in(0, \epsilon)$.

A case of particular importance occurs if $b=0$ and $\inf \operatorname{supp} \sigma \geq 0$. Then $\nu(x) \subseteq(0, \pi)$ and $L \geq \sigma(\{0\})^{-1}$. Thereby $L>\sigma(\{0\})^{-1}$ if and only if $\sigma(\{0\})>0$ and $\int_{0}^{\sigma(\{0\})^{-1}} v(x)^{2} d x<\infty$, and in this case $H(x) \sim \operatorname{diag}(1,0), x \in\left(\sigma(\{0\})^{-1}, L\right)$.

\section{E. Strings}

A string is a pair consisting of a number $L \in[0, \infty]$, and a Borel measure $\mathfrak{m}$ on $\mathbb{R}$ with supp $\mathfrak{m} \subseteq[0, L]$ such that $\mathfrak{m}([0, x])<\infty$ for $x \in[0, L)$ and, in case $L<\infty$, $\mathfrak{m}(\{L\})=0$. We shall denote the string given by $L$ and $\mathfrak{m}$ by $S[L, \mathfrak{m}]$. The number $L$ in $S[L, \mathfrak{m}]$ is referred to as the length of the string.

Define a function $m$ as

$$
m(x):=\mathfrak{m}((-\infty, x)), x \in(-\infty, L) .
$$


Then $m$ is non-decreasing and left-continuous, and we have $m(x)=0$ if $x \leq 0$. Consider the following boundary value problem:

$$
y^{\prime}(x)+\int_{[0, x]} z y(u) d \mathfrak{m}(u)=0, x \in[0, L),
$$

with boundary condition $y^{\prime}(0-)=0$ and, in case $L+m(L)<\infty, y(L)=0$. Thereby $z$ is a complex parameter. Also in this context a notion of TitchmarshWeyl coefficient is of significance: It was shown in [15] that there exist unique solutions $\varphi(x, z)$ and $\psi(x, z)$ of $(2.11)$ which satisfy the initial conditions

$$
\varphi(0, z)=1, \varphi^{\prime}(0-, z)=0, \quad \psi(0, z)=0, \psi^{\prime}(0-, z)=1
$$

and that, for all $z \in \mathbb{C} \backslash[0, \infty)$, the limit

$$
q_{S}(z):=\lim _{x \rightarrow L} \frac{\psi(x, z)}{\varphi(x, z)}
$$

exists. This function is called the Principal Titchmarsh-Weyl coefficient of the string $S[L, \mathfrak{m}]$.

Let $S[L, \mathfrak{m}]$ be a string. Then $q_{S}$ admits a representation

$$
q_{S}(z):=b+\int_{0}^{\infty} \frac{d \sigma_{S}(t)}{t-z},
$$

where $\sigma_{S}$ is some non-negative measure with $\int_{0}^{\infty} \frac{d \sigma_{S}(t)}{1+t}<\infty$, and $b \geq 0$. In fact, $b=\min \operatorname{supp} \mathfrak{m}$. Hence, the Principal Titchmarsh-Weyl coefficient of any string belongs to the Stieltjes class $\mathcal{S}$. [18]:

A basic inverse result going back to M.G.Krein is the following, cf. [17], [6],

Theorem 2.5 (Inverse Spectral Theorem; Strings). The mapping $S[L, \mathfrak{m}] \mapsto q_{S}$ is a bijection of the set of all strings onto the Stieltjes class $\mathcal{S}$.

\section{Inverse spectral relations}

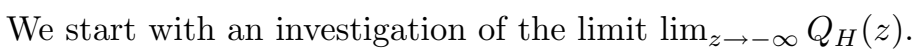

Lemma 3.1. Let $Q \in \mathcal{N}$ and let $a, b, \sigma$ be as in (2.1). Assume that inf $\operatorname{supp} \sigma \geq 0$ and $b=0$. Let $v(x)$ be the (unique) function which corresponds to $Q$ by means of Theorem 2.3, (2.9). Then

$$
\lim _{z \rightarrow-\infty} Q(z)=\lim _{x \searrow 0} v(x) .
$$

Proof. Note that both limits in (3.1) exist in $\mathbb{R} \cup\{-\infty\}$. We show that, for any $a \in \mathbb{R}, \lim _{z \rightarrow-\infty} Q(z)=a$ if and only if $\lim _{x \rightarrow 0} v(x)=a$. Once this is proved, it will also follow that $\lim _{z \rightarrow-\infty} Q(z)=-\infty$ if and only if $\lim _{x \rightarrow 0} v(x)=-\infty$. 
Assume that $\lim _{z \rightarrow-\infty} Q(z)=a \in \mathbb{R}$ and choose $c>-a$. Then the function $Q(z)+c$ is positive on the negative real axis, and hence belongs to the Stieltjes class. It follows that also $z(Q(z)+c) \in \mathcal{N}$ and hence that

$$
Q_{1}(z):=\frac{-1}{z(Q(z)+c)} \in \mathcal{N} .
$$

Clearly, $z Q_{1}(z) \in \mathcal{N}$, and thus $Q_{1} \in \mathcal{S}$. Moreover, $\lim _{z \rightarrow-\infty} Q_{1}(z)=0$. Hence $Q_{1}$ can be represented as $Q_{1}(z)=\int_{[0,+\infty)} \frac{d \tau(\lambda)}{\lambda-z}$, and

$$
\int_{[0,+\infty)} d \tau(\lambda)=-\lim _{z \rightarrow-\infty} z Q_{1}(z)=\frac{1}{a+c} .
$$

Let $S[L, \mathfrak{m}]$ be the (unique) string whose Principal Titchmarsh-Weyl coefficient $q_{S}$ is equal to $Q_{1}$. Then, by [15] and [18],

$$
\lim _{x \searrow 0} m(x)=\left(\int_{[0,+\infty)} d \tau(\lambda)\right)^{-1}=a+c .
$$

Let $H_{1}$ be a Hamiltonian with $Q_{H_{1}}(z)=z Q_{1}(z)$. It was shown in [18] that, if $H_{1}$ is parameterized appropriately, there exists $l>0$ such that

$$
H_{1}(x)=\left(\begin{array}{cc}
1 & -m(x) \\
-m(x) & m(x)^{2}
\end{array}\right), 0 \leq x \leq l .
$$

By (2.6) and (2.8), the Hamiltonian

$$
H_{2}(x):=\left(\begin{array}{cc}
1 & -c \\
0 & 1
\end{array}\right) J H_{1}(x) J^{T}\left(\begin{array}{cc}
1 & 0 \\
-c & 1
\end{array}\right)
$$

has Titchmarsh-Weyl coefficient $Q$. Hence there exists a reparameterization $\lambda$ with $H(x)=H_{2}(\lambda(x)) \lambda^{\prime}(x)$. For $x \in[0, l]$ we have

$$
H_{2}(x)=\left(\begin{array}{cc}
(m(x)-c)^{2} & m(x)-c \\
m(x)-c & 1
\end{array}\right) .
$$

Comparing the right lower corners of $H$ and $H_{2}$ yields that $\left.\lambda\right|_{[0, l]}=\mathrm{id}$, and hence that $v(x)=m(x)-c, x \in[0, l]$. It follows that $\lim _{x \backslash 0} v(x)=a$.

Conversely, if $\lim _{x \backslash 0} v(x)=a$ and $c+a>0$, the function $v(x)+c$ is the mass function of the string with Principal Titchmarsh-Weyl coefficient $Q_{1}$ given by (3.2). According to [15], the fact that $\lim _{x \backslash 0} v(x)+c>0$ implies that $\lim _{z \rightarrow-\infty} Q_{1}(z)=0$, and that the relation (3.3) holds. By the definition of $Q_{1}$, we find $\lim _{z \rightarrow-\infty} Q(z)=a$.

This lemma already has a noteworthy corollary.

Corollary 3.2. Let $Q$ and $v$ be as in Lemma 3.1. Then $Q \in \mathcal{S}$ if and only if $\lim _{x \rightarrow 0} v(x) \geq 0$ In this case $v$ is the mass function of the string whose Principal Titchmarsh-Weyl coefficient is equal to $-(z Q(z))^{-1}$. That is, $v$ is the mass function of the dual string of the string whose Principal Titchmarsh Weyl coefficient is $Q$.

Now we are in position to prove our first main result. 
Theorem 3.3. Let $H$ be a Hamiltonian defined on $[0, L)$ and assume that for some $\epsilon \in(0, L)$ we have

$$
H(x)=\left(\begin{array}{cc}
v(x)^{2} & v(x) \\
v(x) & 1
\end{array}\right), x \in(0, \epsilon),
$$

with a nondecreasing function $v:(0, \epsilon) \rightarrow \mathbb{R}$. Then the $\operatorname{limit} \lim _{y \rightarrow+\infty} Q_{H}(i y)$ exists in $\mathbb{R} \cup\{-\infty\}$ and in fact

$$
\lim _{y \rightarrow+\infty} Q_{H}(i y)=\lim _{x \searrow 0} v(x) .
$$

Proof. Define Hamiltonians $H_{1}$ and $H_{2}$ as

$$
\begin{gathered}
H_{1}(x):= \begin{cases}H(x) & , x \in(0, \epsilon) \\
\operatorname{diag}(1,0) & , x \in[\epsilon,+\infty)\end{cases} \\
H_{2}(x):=H(x+\epsilon), x \in[0, L-\epsilon) .
\end{gathered}
$$

Denote by $W(x, z)$ the transposed of the fundamental matrix solution of the canonical system with Hamiltonian $H$. Then $Q_{H_{1}}(z)=\frac{w_{11}(\epsilon, z)}{w_{21}(\epsilon, z)}$, and $Q$ is given by

$$
Q(z)=\frac{w_{11}(\epsilon, z) Q_{H_{2}}(z)+w_{12}(\epsilon, z)}{w_{21}(\epsilon, z) Q_{H_{2}}(z)+w_{22}(\epsilon, z)} .
$$

A straightforward calculation, using the fact that $\operatorname{det} W(x, z)=1$, will show that

$$
\begin{gathered}
Q(z)-Q_{H_{1}}(z)=-w_{21}(\epsilon, z)^{-2}\left(\frac{w_{22}(\epsilon, z)}{w_{21}(\epsilon, z)}+Q_{H_{2}}(z)\right)^{-1}= \\
=\frac{-1}{z} \cdot\left(\frac{z}{w_{21}(\epsilon, z)}\right)^{2} \cdot \frac{1}{z\left(\frac{w_{22}(\epsilon, z)}{w_{21}(\epsilon, z)}+Q_{H_{2}}(z)\right)}
\end{gathered}
$$

Since $w_{22}(\epsilon, z) w_{21}(\epsilon, z)^{-1}+Q_{H_{2}}(z) \in \mathcal{N}$, the function

$$
f(y):=y \operatorname{Im}\left(\frac{w_{22}(\epsilon, i y)}{w_{21}(\epsilon, i y)}+Q_{H_{2}}(i y)\right)
$$

is nondecreasing for $y>0$. In particular, the last factor in (3.6) is bounded for $z \in$ $i[1, \infty)$. The function $g(z):=z^{-1} w_{12}(\epsilon, z)$ is a real entire function of exponential type, and all its zeros lie in $\mathbb{R} \backslash\{0\}$. Thus its Weierstrass product representation is of the form

$$
g(z)=C e^{A z} \prod\left(1-\frac{z}{z_{n}}\right) e^{z / z_{n}}
$$

where $C$ and $A$ are real constants and $z_{n} \in \mathbb{R}$. Hence $|g(i y)|$ is a nondecreasing function of $y>0$. In particular, the second factor in (3.6) is bounded for $z \in$ $i[1, \infty)$. We see that

$$
\left|Q(i y)-Q_{H_{1}}(i y)\right|=O\left(\frac{1}{y}\right), y \geq 1
$$

This relation, and the fact that $Q_{H_{1}}$ is by Theorem 2.3 analytic on $\mathbb{C} \backslash[0, \infty)$, implies that

$$
\lim _{y \rightarrow+\infty} Q_{H}(i y)=\lim _{y \rightarrow+\infty} Q_{H_{1}}(i y)=\lim _{x \rightarrow-\infty} Q_{H_{1}}(x)
$$


By our definition of $H_{1}$ the function $Q_{H_{1}}$ satisfies the hypothesis of Lemma 3.1, and we conclude that $\lim _{y \rightarrow+\infty} Q_{H}(i y)=\lim _{x \searrow_{0}} v(x)$.

Remark 3.4. Assume that, for some $\epsilon>0$, we have $H(x)=\operatorname{diag}(1,0), x \in(0, \epsilon)$. Then $\lim _{z \rightarrow-\infty} Q_{H}(z)=-\infty$. This tells us that Theorem 3.3 remains true if we, formally, have $v(x)=-\infty$.

As a particular case of Theorem 3.3 we obtain that the assumption inf $\operatorname{supp} \sigma \geq$ 0 in Lemma 3.1 can be relaxed.

Corollary 3.5. Let $Q \in \mathcal{N}$ and let $a, b, \sigma$ be as in (2.1). Assume that inf $\operatorname{supp} \sigma>$ $-\infty$ and $b=0$. Let $v(x)$ be the (unique) function which corresponds to $Q$ by means of Theorem 2.3, (2.9). Then $\lim _{z \rightarrow-\infty} Q(z)=\lim _{x \searrow_{0}} v(x)$.

Proof. According to Theorem 2.3, the assumptions of Theorem 3.3 are satisfied. To establish the present assertion it suffices to note that, since inf $\operatorname{supp} \sigma>-\infty$, the relation $\lim _{y \rightarrow+\infty} Q(i y)=\lim _{z \rightarrow-\infty} Q(z)$ holds.

Corollary 3.6. Let $Q \in \mathcal{N}$ be such that some Hamiltonian $H$ with $Q_{H}=Q$ satisfies the hypothesis of Theorem 3.3. Then $Q \in \mathcal{N}_{1}$ if and only if $\lim _{y \rightarrow+\infty} Q($ iy $) \in \mathbb{R}$.

Proof. Assume that $\lim _{y \rightarrow+\infty} Q(i y)=: a \in \mathbb{R}$. Consider the Hamiltonian $H_{1}$ as in the proof of Theorem 3.3. Then $Q_{H_{1}}-a \in \mathcal{S} \subseteq \mathcal{N}_{1}$, and hence also $Q_{H_{1}} \in \mathcal{N}_{1}$. The relations (3.7) and (2.3) now imply that also $Q \in \mathcal{N}_{1}$. holds.

Note that in general only the implication " $Q \in \mathcal{N}_{1} \Rightarrow \lim _{y \rightarrow+\infty} Q(i y) \in \mathbb{R}$ "

Remark 3.7. The canonical system (1.1) with the boundary condition $y_{1}(0)=$ 0 corresponds to a selfadjoint extension of a symmetric operator with Dirichlet boundary conditions. In [9] the concept of a generalized Friedrichs extension is introduced and characterized by the condition that its $Q$-function does not belong to $\mathcal{N}_{1}$, but $-Q^{-1} \in \mathcal{N}_{1}$. If the assumptions of Theorem 3.3 are satisfied, the condition $\lim _{x \backslash 0} v(x)=-\infty$ characterizes the generalized Friedrichs extension, which is equal to the common Friedrichs extension of semibounded symmetric operators under the assumptions of Corollary 3.5, see [11], [23] for more details.

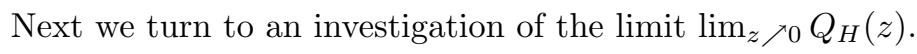

Lemma 3.8. Let $Q \in \mathcal{N}$ and let $a, b, \sigma$ be as in (2.1). Assume that $\inf \operatorname{supp} \sigma \geq 0$, $b=0$, and that $\sigma(\{0\})=0$. Let $v(x)$ be the (unique) function which corresponds to $Q$ by means of Theorem 2.3, (2.9). Then

$$
\lim _{z \nearrow 0} Q(z)=\lim _{x \nearrow L} v(x)
$$

Proof. Note that both limits in (3.8) exist in $\mathbb{R} \cup\{+\infty\}$. Again we shall show that for any $a \in \mathbb{R}$ we have $\lim _{z \nearrow 0} Q(z)=a$ if and only if $\lim _{x} \nearrow_{L} v(x)=a$.

Assume that $\lim _{z \succ 0} Q(z)=a$. Choose $c<-a$, then $Q(x)+c<0$ for $x \in(-\infty, 0)$, and hence $-\frac{1}{Q(z)+c} \in \mathcal{S}$. Thus $Q_{1}(z):=z^{-1}(Q(z)+c) \in \mathcal{N}$. Since, clearly, $z Q_{1}(z) \in \mathcal{N}$, it follows that $Q_{1} \in \mathcal{S}$. 
Let $S[L, \mathfrak{m}]$ be the string with $q_{S}=Q_{1}$. According to [18], the first part of the Hamiltonian corresponding to $Q(z)+z$ is of the form (3.4). Denoting the independent variable in $(3.4)$ by $u$, a scale transformation of the form $x(u)=$ $\int_{[0, u)} m(t)^{2} d t$ brings the first part of the Hamiltonian corresponding to $Q(z)+c$ into the form

$$
\widetilde{H}(x)=\left(\begin{array}{cc}
\widetilde{m}(x)^{-2} & -\widetilde{m}(x)^{-1} \\
-\widetilde{m}(x)^{-1} & 1
\end{array}\right),
$$

with $\widetilde{m}(x)=m(u)$, and it follows that $-\widetilde{m}(x)^{-1}=v(x)+c$. The assumption $\sigma(\{0\})=0$ implies that $v$ is defined on $(0, \infty)$, hence $m(L)=\widetilde{m}(\infty)$, and $L+\int_{[0, L)} m(t)^{2} d t=\infty$. Let $Q_{2}(z)=z Q_{1}\left(z^{2}\right)$. Then, by [18], the trace-normed Hamiltonian $H$ corresponding to $Q_{2}$ is of diagonal form, and the relation $m(L)=$ $\int_{[0,+\infty)}(0,1) H(t)(0,1)^{T} d t$ holds. By $(2.5)$, we have $\int_{[0,+\infty)}(0,1) H(t)(0,1)^{T} d t=$ $-\left(\lim _{y \backslash 0} i y Q_{2}(i y)\right)^{-1}$. Note that $\lim _{y \searrow_{0}} i y Q_{2}(i y)=\lim _{z \succ 0} Q(z)+c$. Summing up, the last relations imply that $\lim _{z \nearrow 0} Q(z)=\lim _{x \nearrow_{L}} v(x)$.

Conversely, assume that $\lim _{x}{ }_{L} v(x)=a$. Again choose $c<-a$, and denote $\widetilde{v}(x)=v(x)+c$. The Hamiltonian corresponding to $Q(z)+c$ is then of the form (2.9) with $\widetilde{v}$ instead of $v$, and a scale transformation of the form $x(u)=\int_{[0, u)} v(t)^{2} d t$ brings it into the form (3.4) with $m(x)=-\widetilde{v}(x)^{-1}$, which implies that $m$ is a mass distribution function of a string. It follows that $Q_{1}(z)=\frac{Q(z)+c}{z}$ is a Stieltjes function, and we find that $\lim _{z \succ 0} Q(z)=a$ by the first part of the proof.

Theorem 3.9. Let $H$ be a Hamiltonian defined on $[0, L)$ and assume that for some $l \in(0, L)$ we have

$$
H(x)=\left(\begin{array}{cc}
v(x)^{2} & v(x) \\
v(x) & 1
\end{array}\right), x \in(l, L),
$$

with a nondecreasing function $v:(l, L) \rightarrow \mathbb{R}$. Then $Q_{H}$ is meromorphic in $\mathbb{C} \backslash$ $[0,+\infty)$, the negative real poles of $Q_{H}$ cannot accumulate at 0 , and the limit $\lim _{z \nearrow 0} Q_{H}(z)$ exists in $\mathbb{R} \cup\{+\infty\}$. In fact we have

$$
\lim _{z \nearrow 0} Q_{H}(z)=\lim _{x \nearrow L} v(x) .
$$

Proof. Consider the Hamiltonian $H_{1}(x):=H(x+l), x \in(0, L-l)$. Let $a_{1}, b_{1}, \sigma_{1}$ be the data in the integral representation of $Q_{H_{1}}$. By Theorems 2.4, 2.3, and Remark $2.2,(i)$, we have $b_{1}=0$ and $\operatorname{supp} \sigma_{1} \in[0, \infty)$. Thus $Q_{H_{1}}$ is analytic in $\mathbb{C} \backslash[0, \infty)$ and the $\operatorname{limit} \lim _{z \nearrow 0} Q_{H_{1}}(z)$ exists in $\mathbb{R} \cup\{+\infty\}$.

If $W$ denotes the transposed of the fundamental matrix solution of the canonical system with Hamiltonian $H$, we have

$$
Q_{H}(z)=\frac{w_{11}(l, z) Q_{H_{1}}(z)+w_{12}(l, z)}{w_{21}(l, z) Q_{H_{1}}(z)+w_{22}(l, z)} .
$$

Hence $Q_{H}$ is meromorphic in $\mathbb{C} \backslash[0, \infty)$ and the $\operatorname{limit}_{\lim _{z} \nearrow_{0}} Q(z)$ exists, in fact $\lim _{z \nearrow 0} Q(z)=\lim _{z \nearrow 0} Q_{H_{1}}(z)$.

Consider the case that $\sigma_{1}(\{0\})=0$. Then $Q_{H_{1}}$ satisfies the assumptions of Lemma 3.8. The relation (3.8) implies together with the last formula that (3.9) 
holds. Assume now that $\sigma_{1}(\{0\})>0$. Then, certainly, $\lim _{z}{ }_{0} Q_{H_{1}}=+\infty$. The relation (2.5) yields that $L<\infty$, and hence, since Weyl's limit point prevails, $\int_{l}^{L} v(x)^{2} d x=\infty$. In particular, $\lim _{x}{ }_{L} v(x)=+\infty$. This shows that also in this case (3.9) holds.

Remark 3.10. Assume that, for some $l<L$, we have $H(x)=\operatorname{diag}(1,0), x \in(l, L)$. Then $\lim _{z \succ 0} Q_{H}(z)=+\infty$. This follows, since in the described situation, we have $Q_{H}(z)=w_{21}(l, z)^{-1} w_{11}(l, z)$, where $W$ is as in the above proof. Hence $Q_{H}$ is meromorphic in $\mathbb{C}$ and has a pole at 0 . This statement just says that the assertion of Theorem 3.9 remains true when we, formally, have $v(x)=+\infty$.

Corollary 3.11. Let $Q \in \mathcal{N}$ and let $a, b, \sigma$ be as in (2.1). Assume that $\operatorname{supp} \sigma \cap$ $(-\infty, 0)$ is a finite set. Let $v(x)$ be the (unique) function which corresponds to $Q$ by means of Theorem 2.3, (2.9). Then $\lim _{z \nearrow 0} Q(z)=-\lim _{x \nearrow L} \cot \nu(x)$, where we understand $\cot \phi=-\infty$ for $\phi \in \pi \mathbb{Z}$.

Proof. By Theorem $2.3 \nu$ is bounded. That is, there are at most finitely many intervals where the Hamiltonian $H$ is of the form diag $(1,0)$, and there are at most finitely many points where $v$ has a negative jump or becomes singular. By (2.5), $\int_{(0, L)}(0,1) H(t)(0,1)^{T} d t=\sigma(\{0\})^{-1}$. If $\sigma(\{0\})=0$, then $L=+\infty$, and $v$ is nondecreasing on some interval $(l,+\infty)$. Hence, the assumptions of Theorem 3.9 are satisfied. If $\sigma(\{0\})>0$, then either $L<+\infty$ and there is some $l<L$ such that $v$ is nondecreasing on $(l, L)$ and $\int_{(l, L)} v(x)^{2} d x=+\infty$, that is, $v(L-)=-\cot \nu(L-)=+\infty$, or $H=\operatorname{diag}(1,0)$ on some interval $\left(l_{0},+\infty\right)$, that is $-\cot \nu(L-)=+\infty$ on $\left(l_{0},+\infty\right)$. Clearly, if $\sigma(\{0\})>0$ then $Q(0-)=+\infty$.

\section{References}

1. V.I.Arnold, Mathematical methods of classical physics, Springer, New York, 1989.

2. N.Aronszajn, W.Donoghue, "On the exponential representations of analytic functions in the upper half plane with positive imaginary part", J.Analyse Math. 5 (1956/57), $321-388$.

3. F.V.Atkinson, Discrete and continuous boundary problems, Academic Press, New York 1964.

4. L. de Branges, Hilbert spaces of entire functions, Prentice Hall, Englewood Cliffs, N.J., 1968.

5. V.Derkach, M.Malamud, "On some classes of holomorphic operator functions with nonnegative imaginary part", 16th OT Proceedings (1997), 113-147.

6. H. Dym and H.P. McKean, Gaussian Processes, Function Theory, and the Inverse Spectral Problem. Academic Press, New York, 1976.

7. H.Flanders, Differential forms with applications to the physical sciences, Dover Publ., New York, 1989.

8. I.Gohberg, M.G.Krein, Theory and applications of Volterra operators in Hilbert space, Translations of Mathematical Monographs, AMS, Providence, Rhode Island, 1970. 
9. S. Hassi, H. Langer, and H.S.V. de Snoo, "Selfadjoint extensions for a class of symmetric operators with defect numbers (1,1)", 15th OT Conference Proceedings, (1995), $115-145$.

10. S. Hassi, H.S.V. de Snoo, "On some subclasses of Nevanlinna functions", Z. Analysis und ihre Anwendungen 15 (1996), 1-11.

11. S. Hassi, H.S.V. de Snoo, and A.D.I. Willemsma, "Smooth rank one perturbations of selfadjoint operators", Proc. Amer. Math. Soc., 126 (1998), 2663-2675.

12. S. Hassi, H.S.V. de Snoo, and H. Winkler, "Boundary-value problems for twodimensional canonical systems", Integral Equations Oper. Theory, 36 (2000), 445479 .

13. I.S.Kac, "On integral representations of analytic functions mapping the upper half plane onto a part of itself", Uspeki Mat. Nauk 11 (1956), 139-144.

14. I.S. Kac and M.G. Krein, " $R$-functions-analytic functions mapping the upper halfplane into itself", Supplement II to the Russian edition of F.V. Atkinson, Discrete and continuous boundary problems, Mir, Moscow, 1968 (Russian) (English translation: Amer. Math. Soc. Transl., (2) 103 (1974), 1-18.

15. I.S. Kac and M.G. Krein, "On the spectral functions of the string", Supplement II to the Russian edition of F.V. Atkinson, Discrete and continuous boundary problems, Mir, Moscow, 1968 (Russian) (English translation: Amer. Math. Soc. Transl., (2) 103 (1974), 19-102).

16. M.Kaltenbäck, H.Winkler, H.Woracek, "Generalized Nevanlinna functions with essentially positive spectrum", J.Oper.Theory, to appear.

17. M.G.Krein, "On inverse problems for the nonhomogeneous cord", Dokl.Akad.Nauk 82 (1952), 669-672 (Russian).

18. H. Langer and H. Winkler, "Direct and inverse spectral problems for generalized strings", Integral Equations Oper. Theory 30 (1998), 409 - 431.

19. T.J.Stieltjes, Euvres completes/Collected papers. Vol. I, II, reprint of the 1914-1918 edition. Springer-Verlag, Berlin 1993.

20. H. Winkler, "The inverse spectral problem for canonical systems", Integral Equations Oper. Theory, 22 (1995), 360-374.

21. H. Winkler, "On transformations of canonical systems", Oper. Theory Adv. Appl. 80 (1995), 276 - 288.

22. H. Winkler, "Canonical systems with a semibounded spectrum", Oper. Theory Adv. Appl. 106 (1998), 397 - 417.

23. H. Winkler, "On generalized Friedrichs and Krĕn-von Neumann extensions and canonical systems", Math. Nachr. 236 (2002), 175 - 191.

Henrik Winkler

Institut für Mathematik, MA 6-4

Technische Universität Berlin

Straße des 17. Juni 136

D-10623 Berlin

Germany

e-mail: winkler@math.tu-berlin.de 
Harald Woracek

Institut für Analysis und Scientific Computing

Technische Universität Wien

Wiedner Hauptstr. 8-10/101

A-1040 Wien

Austria

e-mail: harald. woracek@tuwien.ac.at 